\title{
Techniques of geomatics and soft computing for the monitoring of infrastructures and the management of big data
}

\author{
ERnesto Bernardo $^{1 *}$, Stefano Bonfa ${ }^{2}$, SAlvatore CAlCAGnO ${ }^{1}$ \\ ${ }^{1}$ DICEAM - Civil, Energy Environmental and Material Engineering Department \\ Mediterranea University \\ Località Feo di Vito, 89124 Reggio Calabria, ITALY \\ ${ }^{2}$ UK Economic Interest Grouping (UKEIG) N. GEE000176 \\ 87 Hungerdown E4 6QJ London \\ UNITED KINGDOM
}

\begin{abstract}
The proposed research activity is based on the study and development of advanced survey and monitoring techniques for the control and mapping of road infrastructures. Specifically, we want to create an automated monitoring system mainly through the use of drones that at pre-established time steps acquire the data necessary for the continuous monitoring of the functional characteristics of the road infrastructure and the public usability of dynamic data. Subsequently, through the implementation of algorithms dedicated to the management of the amount of georeferenced data acquired - big data - the same will be represented on GIS (Geographic Information System) platforms as "open and updatable" thematic cartography, which can be integrated with further data collected both with of traditional Geomatics (GNSS receivers, motorized total station and 3D laser scanner) and innovative ones (remote sensing, Mobile Mapping Systems (road vehicles and UAVs)). This context also includes the establishment and updating of the Road Cadastre, introduced by the Ministerial Decree of $01 / 06 / 2001$ No. 6 , intended as an IT tool for archiving, viewing, querying and managing all the data that the body owner / manager owns on its own road network.
\end{abstract}

Key-Words: - UAV, Image classification, Big Data, GIS, Image analysis, Segmentation, Road Cadastre

Received: November 23, 2020. Revised: April 1, 2021. Accepted: April 12, 2021. Published: April 22, 2021.

\section{Introduction}

The thematic area of the proposed research relates to the area of specialization (indicated by the National Strategy of Intelligent Specialization) of Sustainable Mobility, a tool for reducing the economic and social impact on the transport system in the near future.

The objective of developing sustainable, reliable and safe transport systems can be made feasible thanks to the development and dissemination of the "Intelligent Transport Systems (ITS)".

In addition, the "Smart Road" project is being developed in Europe, which makes it possible to move from the classic concept of road, intended as a mere civil infrastructure connecting two points, to the more modern one of a so-called fully integrated "communication road" in the intermodality network. It appears to be a project with a high rate of innovation in the road sector and has as its fulcrum the user and his protection, to be obtained above all through the continuous monitoring of the functional characteristics of the road infrastructure (by means of new generation robotic and automated piloted devices remote) and the public usability of dynamic data acquired in the form of "open data" and "big data".
One of the aims of the research was also the design and construction of specific measurement components / sensors to be equipped on remotely piloted technological and motorized systems for road applications and the possible representation of the elements inherent to the geometric characteristics of the roads and related appurtenances, as well as permanent installations and services connected to the needs of traffic through the creation of a database that allows rapid selective searches by topics.

The geomatics methodologies, combined with the use of technologies and sensors aimed at the complete digitization of the characteristics of the object under investigation and their management (big data), could be useful for organizations / managers, companies and users.

The proposed research is part of the group of interventions aimed at developing "smart communities", in which the "physical infrastructures" are equipped with "technological and digital infrastructures" in order to have an exchange of information blocks between users and objects, in order to improve the lives of citizens and the business of companies, in terms of safety and social and economic impact. 
In this sector, the integration of big data (as a result of data acquisition following detection activities using automated, robotic and remote piloted technological systems) and GIS is to be considered the most adequate, sustainable and in some way indispensable tool to make structured geographical information is homogeneous and transferable, including interoperability and above all the circulation of data on the web.

Furthermore, it will be possible for users to interact with GIS updates.

\section{Materials and Methods}

GIS update used for road cadastre - proposed automatic update methodology.

1. We tested an innovative procedure that involves the use of a suitably designed fleet of drones, which, proceeding from point a to point $b$, allows to acquire images of the object of study.

2. Subsequently, the images are processed by special automated algorithms aimed at identifying holes, road signs, traffic lights, manholes and their subsequent display and updating of a basic cartography on the GIS system.

3. The acquired data are therefore high-resolution images with high spatial sampling rates.

In this research activity we focused specifically on the analysis of the attributes in the database that can be identified through the classification and segmentation of the images acquired by the proposed system: presence of degradation of the road surface, presence and maintenance status of horizontal and vertical signs and presence of manholes.

\section{Innovative measuring system}

We used a fleet of automated drones connected to the cloud (or to a local network) which are automatically recharged through special charging stations located at pre-established points.

The drone fleet sends data in real time to the cloud platform, which is subsequently processed by the algorithms to select the images.

The data acquisition system provides for the installation of two platforms along the path to be monitored to allow the drone battery to be recharged and the data necessary for subsequent processing to be transferred.

We have built an innovative monitoring system including drones, intelligent multi-landing and charging pads, automatically governed that communicate at short range with nearby drones and indicate the status of the station (Fig. 1).
1. When a drone in flight detects that the battery is running low, it looks for the nearest charging station, the latter communicates to the drone if there is a free pitch and a charged battery available.

2. Having obtained the ok to land, the drone, knowing the GPS coordinates of the station, approaches and, moving vertically, lands on the assigned stand.

3. Once landed, a subsystem recharges the onboard battery or swaps it, replacing the discharged battery with a charged one. During the replacement, the drone is still powered through a special connector in order not to lose communications and allow the automatic procedure with the exchange of information. After recharging, take-off takes place.

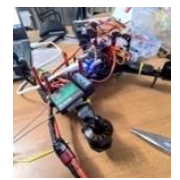

(a)

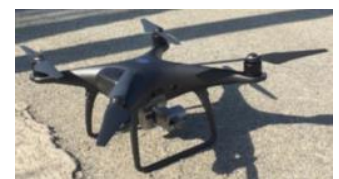

(b)

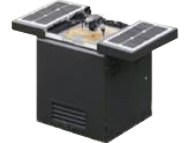

(c)
Fig. 1. (a) Automated drone system components, (b) UAV Phantom 4 Pro, (c) Drone input/output wireless charging base.

The wireless charging station is made up of an "intelligent" induction plate which, when the drone lands, determines the type of batteries supplied to the aircraft, and thus establishes the correct charging parameters.

This is made possible thanks to a small device on board the drone consisting of a microcircuit with a data transmission system, weighing a few tens of grams and dimensions contained in the order of a few centimeters, such that it can be installed not only on large professional drones but also on smaller commercial ones.

These stations are totally waterproof and weatherproof and also serve as a temporary shelter for appliances.

In particular, the process of this first experimentation is divided into three automated phases, schematized in the flow diagram in Fig. 2 - 3 and which include:

1. Definition of the flight plan, in terms of Ground Sampling Distance (GDS), overlapping of images and waypoints.

2. Image analysis: preprocessing - segmentation classification. In order to improve the precision of the image processing and the identification of the investigated element, the use of different 
cascading algorithms was experimented which involve the combination of different methodologies (segregation; edge detector; Canny filter; Gaussian filter; Support Vector Machine - SVM).

3. Geo-localization of data on the GIS platform to associate the coordinates of the data implemented in the database to each element of the GIS.

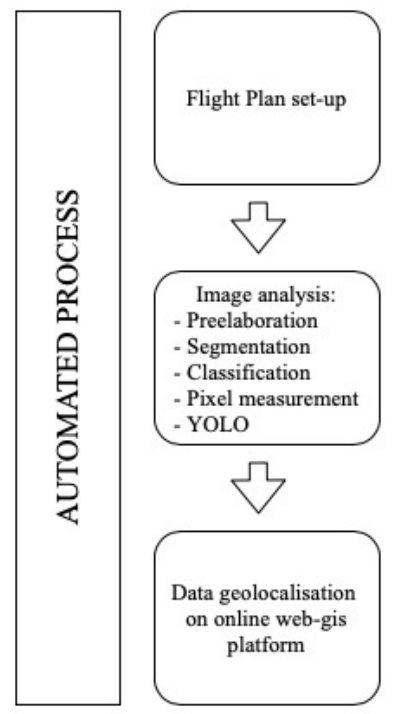

Fig. 1. Workflow for road safety assessment.

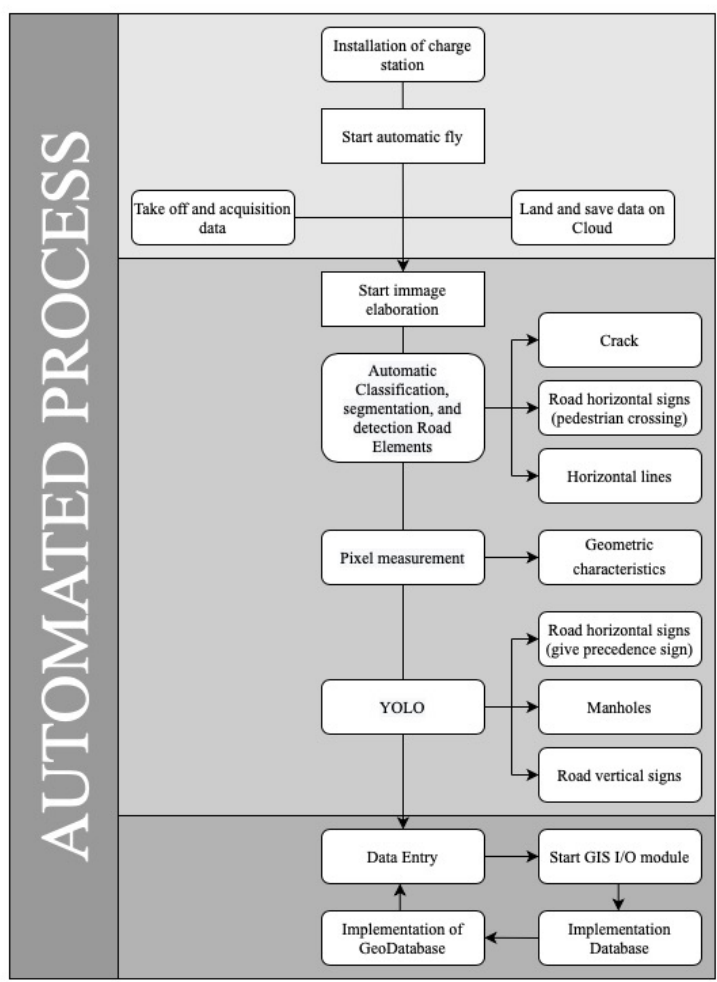

Fig. 3. Workflow automatic system Gis integration. The interventions were tested on a very important road in the territory of the city of Messina (ME),
Sicily, viale Europa, (Fig. 4) and the tests were performed at different flight heights based on the defined flight plan.

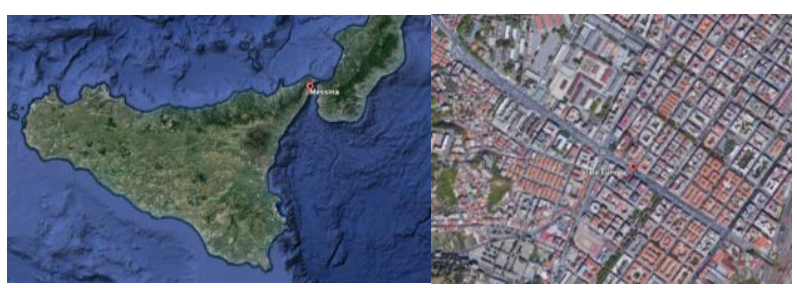

Fig. 4. Test area; Viale Europa, Messina, (ME), Sicilia, Italia.

\subsection{Flight plan set-up}

We detected a most important road in the territory of the city of Messina and the surveys were carried out in a time with little traffic.

For the acquisition phase of the photographic data set, the DJI Mavic 2 Pro UAV was used. To obtain images with high-quality centimeter accuracy, the image acquisition plan was divided into three phases:

1) Definition of the type of plan of image acquisition;

2) Definition of Ground Sampling Distance (GSD);

3) Definition of image overlay. The type of image capture was set on the automatic waypoint flight mission for the UAV to perform an automatic flight.

The way points have been positioned along the road development. The software automatically calculates the image acquisition plan and the mission settings, has defined the following parameters: flight height (consequently GSD), overlap (\%) and area to be mapped. To obtain centimeter accuracy $(\mathrm{GSD}<1)$ the legal flight height, considering the specifications of the DJI Mavic 2 Pro camera the maximum flight height has been set to 10 meters for vertical flight. Several missions along the way were performed automatically using the Pix 4d flight planning software (Pix4d, Lausanne). To collect photographic information, flight plans were organized with the camera inclination set to $70^{\circ}$. During the flight, the drone has always maintained a constant height with respect to the take-off point, therefore initial tests were necessary to verify that the difference in height could not in any way compromise the safety of flight operations. We also proceeded to verify the absence of any obstacles (electrical cables or subways) along the flight plan. Apps like Flight plan, however, allow us to change the various heights of the way points thus optimizing the procedures necessary for flight. 


\subsection{Image analysis}

In order to improve the quality and precision of the images, particular attention was paid to the postprocessing procedure of the images, experimenting with the use of different ad hoc algorithms that involve the combination of various methodologies: Preelaboration (Sobel and Prewit operator), Segmentation (Edge detector, Canny filter, Gaussian filter) and Classification (SVM). All this, in order to optimize the quality of the image, perform its segmentation and its final classification.

In particular, edge detector is used to mark the points of a digital image where the light intensity changes abruptly. As known, images are the representation of the physical world, sudden changes in the properties of an image are the symptom of important events or changes in the physical world. These changes can be for example: discontinuity of depth, discontinuity of the orientation of the surfaces, modification of the properties of the materials, and variations of the illumination coming from the surrounding environment.

Moreover, in the field of research of image treatment and artificial vision, in particular of the feature extraction branch, the recognition of the contours plays a fundamental role.

The contour recognition operation generates images containing much less information than the original ones, since it eliminates most of the details that are not relevant for the identification of the contours, while retaining the essential information to describe the shape and the structural and geometric characteristics of the objects represented.

In order to improve the quality of the images and the information they contain, multiple algorithms have been used to improve the recognition of the contours of the holes in the image and thus avoid any attribution of deformation of the road to shadows or other noise elements.

The algorithms used were edge detectors, canny and gaussian filters.

Clearly, a Pre-processing has been made previously. Captured images have been converted to grayscale; those with uneven background lighting have been corrected to achieve the same results as evenly lit ones. Preprocessing was based on removing pixels in the image that have an average intensity compared to higher-intensity pixels. In order to improve the image, an attempt was made to eliminate the noise in the image in question through the use of two operators: Sobel and Prewitt (Fig. 5). This procedure is used as early cracks' detection.
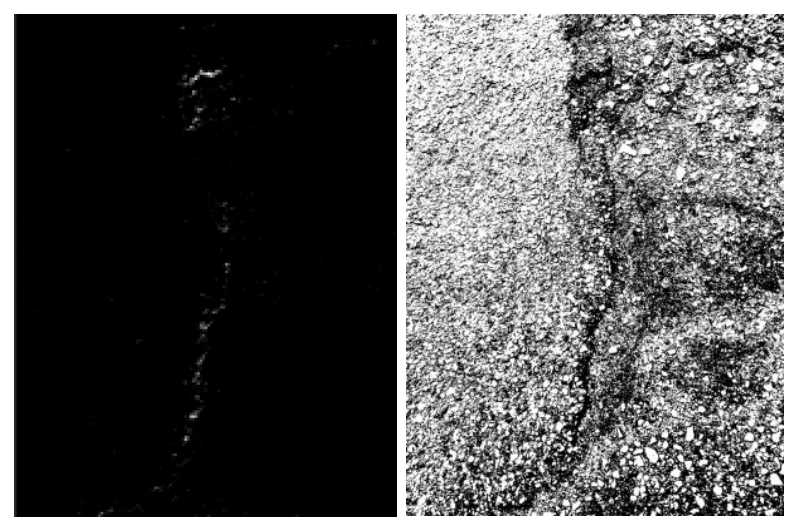

Fig. 5. Pre-elaboration with Sobel e Prewitt

Regarding the edge detector, the detection of the edge is achieved by maximizing the signal-to-noise ratio (of the amplitudes) of the gradient. The expression used for the $\mathrm{s} / \mathrm{r}$ ratio is:

$s n r=\frac{h_{e}}{\sigma_{n}} S(h), \operatorname{con} S(h)=\frac{\int_{-w}^{0} h(x) d x}{\int_{-w}^{w}[h(x)]^{2} d x}$

Where $h_{e}$ is the amplitude of the step while $\sigma_{n}$ is the standard deviation of the noise.

Points recognized as edge should be as close as possible to the center of the actual edge. For this purpose, a localization factor is defined ( $h$ ' is the derivative of $h$ ):

$L O C=\frac{h_{e}}{\sigma_{n}} L(h), \operatorname{con} L(h)=\frac{h^{\prime}(0)}{\int_{-w}^{w}\left[h^{\prime}(x)\right]^{2} d x}$

As for the uniqueness of the operator's response, the $\mathrm{x}_{\mathrm{m}}$ distance between two gradient peaks, in the presence of only noise, is supposed to be equal to a k-fraction of the operator's W width:

$$
\mathrm{x}_{\mathrm{m}}=\mathrm{Kw}
$$

The image of response to the operator, when viewed as a 3D surface, is characterized by valleys and reliefs. The maximum curves of the reliefs are called ridges. To get a unique response from the edge detector, we need an algorithm that suppresses all multiple responses, that is, pixels that have high response values to the operator but are not local maximums for it.

The non-maximum suppression is obtained by searching for pixels that are maximum for the magnitude along the direction of the gradient. Only ridges are held by suppressing all other points that are not at the local maximum height. 
The quality of the results obtained with the Canny method, higher than that of all other gradient operators, is justified by the fact that the method uses two thresholds, one for the detection of the sharpest edges, the other for the detection of the edges weaker. However, these are only taken into account if they are connected to net.

As for the use of the Cunny method, we can get the image in a grayscale that was smoothed for the first time using a Gaussian filter with a specific standard deviation aimed at reducing noise (example shown in Figure 6), and after that they were gradients for determining the points of the border [1]. We use two threshold values 0.4 (Figure 6.a) and 0.5 (Figure 6.b), with several border points that have been related together. The strong point of the method makes it possible to detect edges even when there is noise and at the same time to detect weak edges. Early margin detection results found that the disage came from the parameters used in the algorithm and that the optimal parameters varied in the wake of each image.

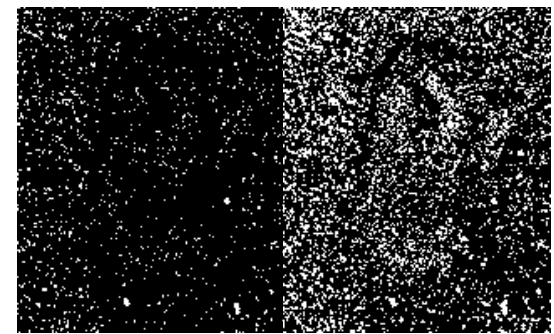

Fig. 6. Processing: a) threshold value $=0.4$, b) threshold value $=0,5$

In the Segmentation phase, following the cracks detection (Fig. 7.a) an image segmentation tasks were performed: the process was used to partitioning the image into disjointed and homogeneous regions. The purpose of segmentation is to go to simplify and/or change the representation of images to something that is easier to analyze. It is usually used to detect objects and borders (lines, curves, etc.) (Fig. 7.b) but more precisely it is seen as the process by which the pixels of the image are classified that have common characteristics, so each pixel in a region is similar to others in the same region for common characteristics (color, intensity or texture). As the noise in the image increases, the segmentation process becomes more and more complex, and in addition, an uneven light source can make the success of the process more articulated. In this case, the best results were obtained:

- With more sophisticated (nonlinear) algorithms for calculating the size of the gradient (sum of the square of the response of a horizontal edge finder and the square of the response of a vertical edge finder);

- With smarter strategies (Canny algorithm) (Fig. 7.c).

Canny's method is an excellent edge detector among the traditional algorithms that are on the market. A border is identified by the relationship between the image and the filter. The filter is chosen on the basis of three effectiveness criteria defined by Canny:

- Good detection capability: The operator has a low probability of not detecting a real edge and a low probability of spotting false edges;

- Good tracking ability: The points highlighted by the operator should be as close as possible to the center of the real edge;

- Uniqueness of response: operator should provide only one answer at a real edge.

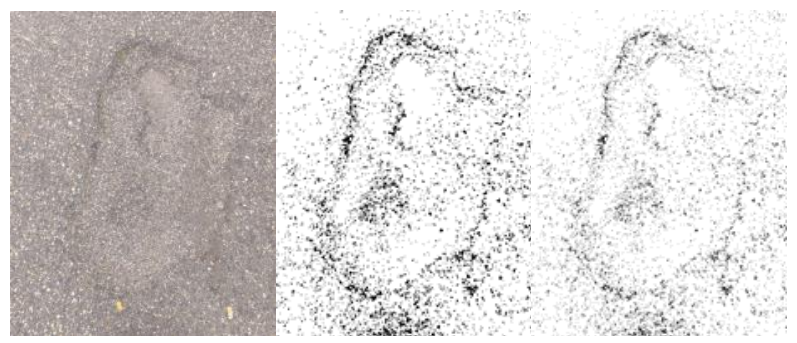

Fig. 7. Preprocessing: a) none; b) edge detector; c) Canny filter

Finally, Classification has been carried out with the SVM method.

In the machine learning sector, support-vector machines (SVM) are supervised learning models with associated learning algorithms that analyze data used for classification and regression analysis.

It is popular in applications such as natural language processing, speech and image recognition and computer vision but achieves maximum effectiveness in binary classification problems.

The SVM is based on the idea of finding a hyperplane that best divides a dataset into two classes.

To do this, perform the following steps:

1) Look for a linearly separable hyperplane or a decision limit that separates the values of one class from another. (If there is more than one, look for the one that has the highest margin with the support vectors, to improve the accuracy of the model);

2) If such a hyperplane does not exist, SVM uses a nonlinear mapping to transform the training data into a higher dimension (if we are in two dimensions, it will evaluate the data in 3 dimensions). In this way, the data of two classes can always be separated by a hyperplane, which will be chosen for the division of the data. 
Compared with other methods such as artificial neural network, SVMs have significant advantages of high accuracy, elegant mathematical tractability, and direct geometric interpretation [2-9].

We tested four SVMs with different kernels (LIN, HPOL, IPOL, and GRB). In the case of using linear kernel, the KSVM degrades to original linear SVM. We computed hundreds of simulations in order to estimate the optimal parameters of the kernel functions, such as "the order d" in HPOL and IPOL kernel, and "the scaling factor $\gamma$ " in GRB kernel. The comparison was made through the confusion matrices in which the element of $i^{\text {th }}$ row and $j^{\text {th }}$ column represents the classification accuracy belonging to class $i$ are assigned to class $j$ after the classification $[10 ; 11]$.

In our case, the results showed that the proposed DWT + PCA + KSVM method obtains quite excellent results on both training and validation images. For LIN kernel, the whole classification accuracy was (15 $+137) / 160=95 \%$; for HPOL kernel, was $(17+$ $138) / 160=96.88 \%$; for IPOL kernel, was $(16+$ $141) / 160=98.12 \%$; and for the GRB kernel, was $(18$ $+141) / 160=99.38 \%$. Obviously, the GRB kernel SVM outperformed the other three kernel SVMs.

The comparison results indicate that the used method DWT+PCA+KSVM with GRB kernel performed best, achieving the best classification accuracy as $99.38 \%$.

Computation time is another important factor to evaluate the classifier.

The time for SVM training was not considered, since the parameters of the SVM keep unchanged after training. We sent all the 160 images into the classifier, recorded corresponding computation time, computed the average value, depicted consumed time of different stages.

For each image, the averaged computation time on feature extraction, feature reduction, and SVM classification is $0.092 \mathrm{~s}, 0.0748 \mathrm{~s}$, and $0.0124 \mathrm{~s}$, respectively. The feature extraction stage is the most time-consuming as $0.092 \mathrm{~s}$. The feature reduction costs $0.0748 \mathrm{~s}$. The SVM classification costs the least time only $0.0124 \mathrm{~s}$. The total computation time for each image is about $0.1792 \mathrm{~s}$, which is rapid enough for a real time analysis.

Referring to the study area previously exposed, using the method described in [34], the sequence shown in Fig. 8 represents the logical application process referred to in the previous paragraph (further improved step by step thanks to the use of particular local filters and morphological operators) to identify different types of crack that are usually present in common roads (alligator, longitudinal, transverse, non-crack) the cracks. Crack and non-crack regions were extracted from background images using the image processing techniques and methodologies above discussed. The results of this processing are provided in Fig. 8. This figure shows the image for all kind of cracks (alligator, longitudinal, transverse, non-crack), Fig. 8a input image shows the input images captured by the UAV. All images have been resized to $512 \times 512$ pixels to speed up algorithm processing time. The images with non-uniform lighting were adjusted through a morphological operation with a disk-shaped filter.

After the application of the above procedures, the results of which are shown in Fig. 8b, the images were then processed using both segmentation methodologies (edge detector e Canny) and automatically threshold value. The median filter with a rectangular element of $3 \times 3$ pixels was then applied, and regions with areas greater than 500 pixels were removed. Finally, the structures of the potential crack regions were enhanced through a morphological closure operation with a diamond-shaped filter with a radius of $5 \times 5$ pixels. The final shape of the potential cracks can be seen in the last figure (Fig. 8c). In post processing we proceed to enhance images removing the noise (Fig. 8d) and proceed to detect crack candidates (Fig. 8e) [12 - 19].
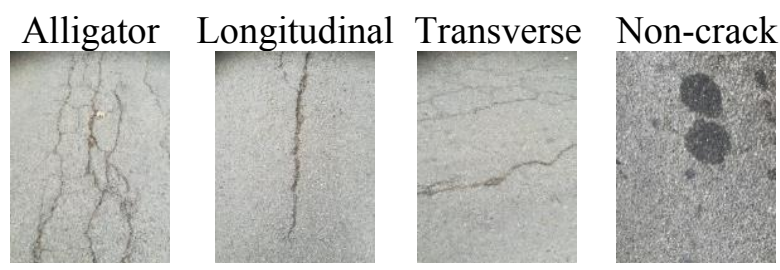

(a) Input Images
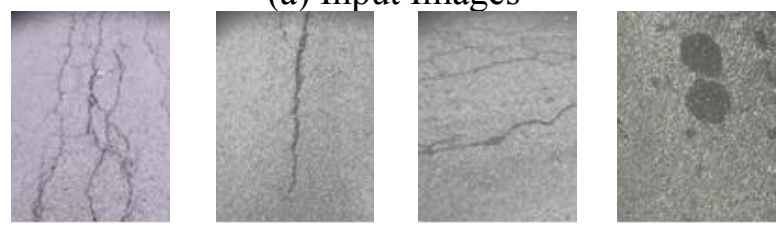

(b) Illumination Correction
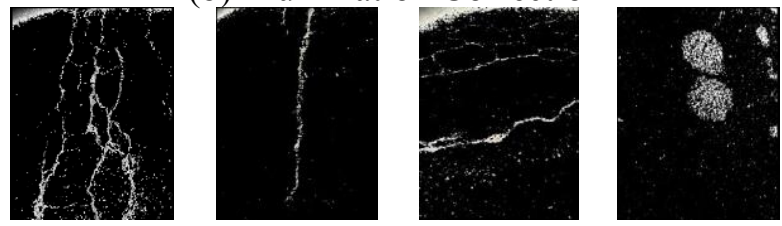

(c) Thresholding based Segmentation
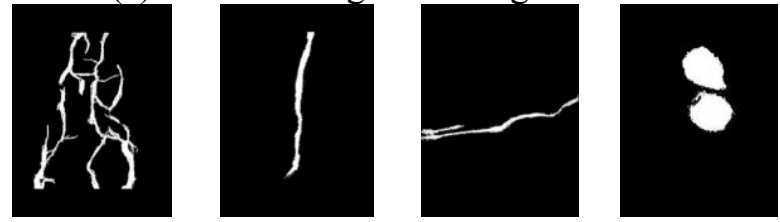

(d) Post Processing and Image Enhancement 

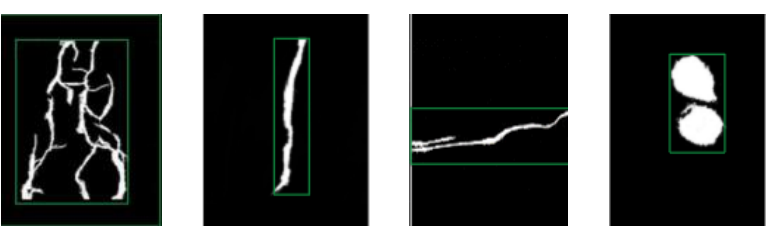

(e) Detected Crack Candidates

Fig.8. Process crack detection

After applying the image analysis techniques to segment and improve the edges and outlines of the images, the crack detection application was carried out through the use of the SVM.

The classification with SVM was carried out in two phases:

1) SVM Training;

2) Performance testing.

In the first phase, the geometric characteristics of the linked components assigned for SVM training were initially calculated. Then, these features were normalized to a range $(0,1)$. Kernel with radial base function (RBF) was chosen as a kernel trick, because the number of instances (connected regions) was not very large and the size of the space transformed with RBF is infinite. The optimal training parameters for the SVM were found using grid search. During this operation, triple cross-validation was performed to correctly learn the different types of cracks. In this triple cross-validation, the training set was divided into 3 equal subsets. To ensure proper learning, a subset was tested using the trained classifier on the remaining two subsets. The goal was to identify good parameters so that the classifier can predict test data effectively. After learning that the parameters had been determined, SVM was trained with the "One Against All" approach using the MATLAB LIBSVM library.

In the second part, connected regions were tested that were not used during SVM training. The results of the classification have been tabulated in Table 1 .

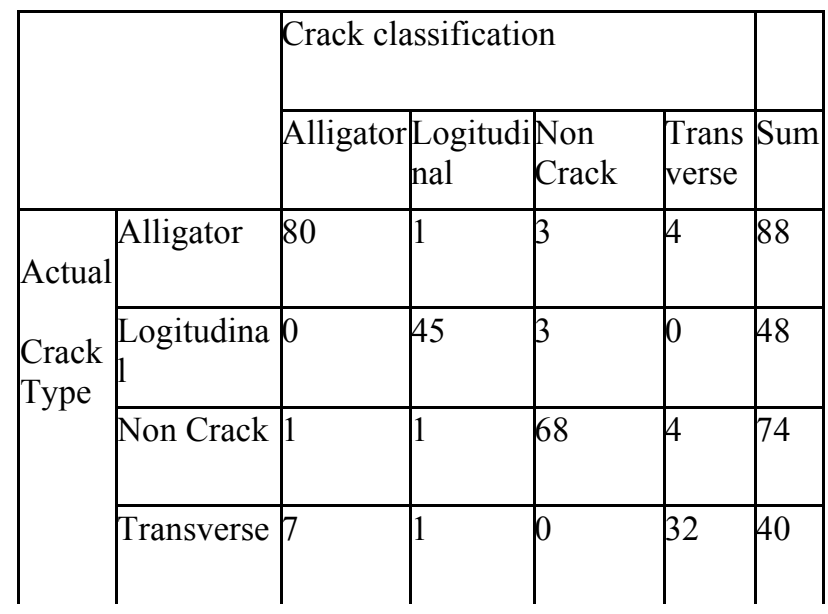

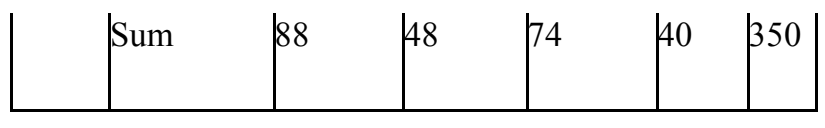

Table 1. Results

In this table, the number "64" indicates that 64 regions of potential crack were correctly classified, meaning there were actually alligator cracks and were considered as such. On the other hand, "12" represents the number of alligator cracks that have been misclassified as longitudinal fissures, and so on.

The results of the classification showed that the proposed algorithm has a higher capacity in terms of classification of longitudinal, transverse and noncracked cracks, but the classification of alligator cracks did not have the same because of their complex and variable forms. In fact, some alligatorshaped cracks have been misclassified as other types of cracks, this is mainly due to parts disjointed compared to the rest of the main body of the crack.

Clearly, the high number of the shapes of cracks present in road infrastructure does not always allow to use the proposed procedure and often requires the implementation of neural networks with different layers and many neurons, thus having a considerable "degradation" of the performance of the software to be implemented [20]. Therefore, it was decided to adopt a modular approach: to use multiple neural networks, each of which recognizes not the whole crack but a part of it. This first bottom-up approach is followed by another top-down one, during which, through a deterministic function, the various recognized parts are analyzed, and the object is identified which is unequivocally composed of the parts themselves. The supervised elements are considered a set of "border elements" (cracks) of "color domains" of the border, of "image contained in the border" (internal image). In turn, the "border elements" are considered a composition of predefined "geometric elements"; it is evident that a given "primitive" element, that is not broken down into other elements, can belong to several supervised symbols (those to be recognized).

\subsection{Determination of geometric characteristics through pixel measurement}

The geometric characteristics of the road, of the artefacts and of the deterioration are obtained directly from the frames having fixed a correction plane and the focal point of the camera (Fig. 9). 


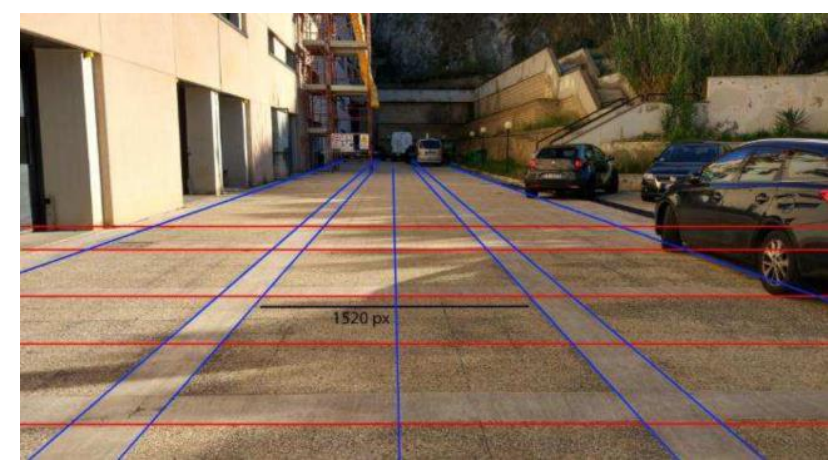

Fig. 9. Correction plane (grid) on slope 0.

Segments are automatically plotted on frames starting from the reference axes and applied two scaling functions to determine the length and width. More specifically, in order to determine the length function, different measurements were made in pixels of sample objects of known dimensions at different distances from the pick-up point, the results obtained were then analyzed and approximated in a single function:

$$
y=0.0005167 \cdot x^{2}
$$

In which is expressed the function of scale for length experimentally obtained.

$$
\mathrm{X}=\frac{\text { Distance between the axes }}{n^{\circ} \text { pixel between axes }} \cdot \mathrm{n}^{\circ} \text { pixel object to measured }
$$

In which is the function of scale by width.

In the example (Fig. 10) shown the dimensions of the manhole obtained by the two functions are respectively 0.705 meters and 0.696 meters, reporting an error therefore in the order of half a centimeter. The grid was subsequently adapted according to the slope of the road.

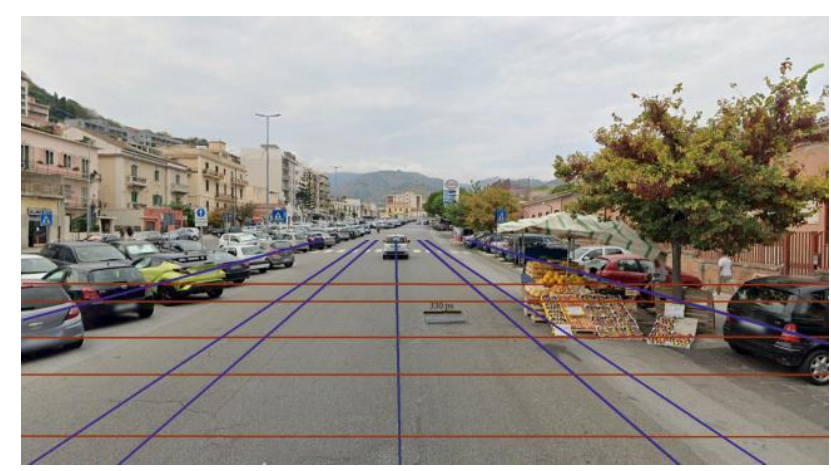

Fig. 10. Example road artifact measures (manhole).

\subsection{Acquisition of the frame of the artifacts, signs and signals (Object detection - Pattern Recognition)}

Other data were also collected with the Mobile Mapping System as well as with UAVs.

Object Recognition in the Computer Vision is the ability to find a determined object in a sequence of images or videos. You Only Look Once (YOLO) is an advanced object recognition system developed within the Object Recognition. This method, programmed by Joseph Redmond, a mathematician and computer scientist at the University of Washington, is much faster than traditional artificial intelligence systems.

The above-mentioned methodology (used for the implementation of the system proposed in this note), uses a single neural network. For the recognition of vertical signage, this neural network provides for bounding boxes and the probabilities of associating the pixels of the image to a class directly from the complete images (avoiding the partition of the image) in a single evaluation for the recognition of vertical signage.

Because the entire detection pipeline is a single network, it can be optimized end-to- end directly on detection performance. The unified architecture is extremely fast. The YOLO basic model processes images in real time at 45 frames per second and is well suited for use on MMS for real time detection, in movement, of vertical signs and artefacts.

In this regard, we report an example of the application of the methodology inherent in the recognition of different signals and manholes acquired with the method described above acquired both with the MMS in circulation along the Viale Europa in Messina (Fig. 11-12) and with the UAVs in flight on the same road (Fig.13).

YOLO reframes object detection as a single regression problem, straight from the image.

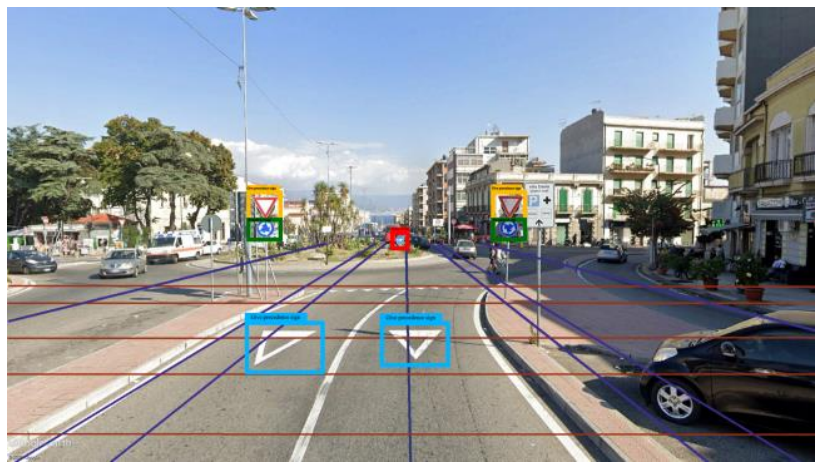

Fig. 11. Example of object recognition from MMS. 


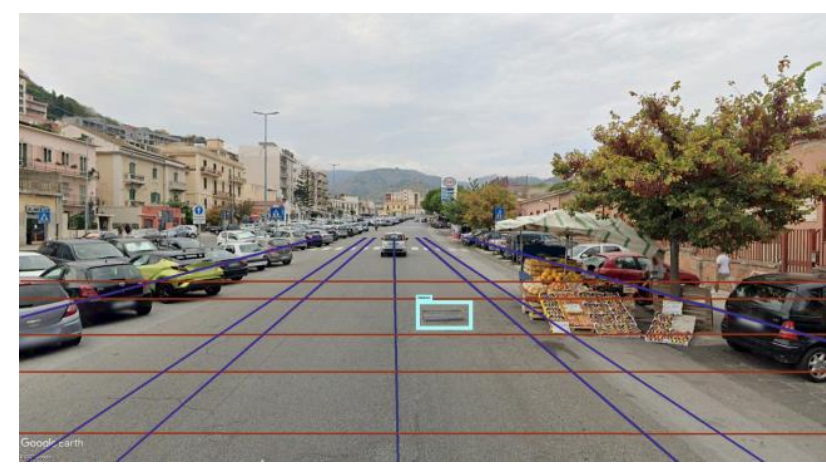

Fig.12. Example of object recognition from UAV (manhole).

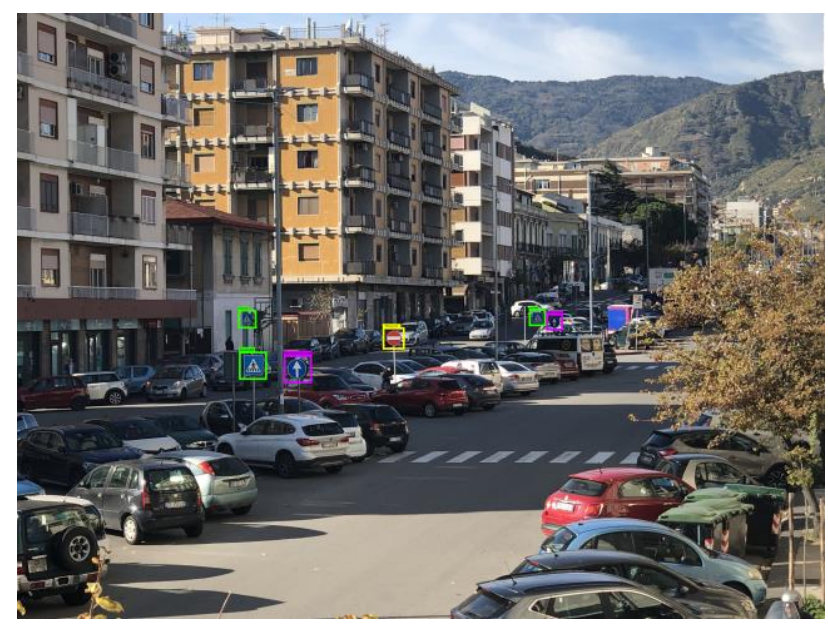

Fig.13. Example of object recognition from UAV.

The immediate restitution of the data is well suited to be used as an integral part of a system dedicated to updating a Road Cadastre.

The proposed system identifies the elements of interest, memorizing the frame by acquiring it at a given distance and georeferencing it according to the diagram shown in the figure.

In particular, the distance is determined in relation to the number of pixels of the recognized object (the signal), having the known dimensions. In the system calibration phase, in fact, several frames of the same signal were acquired at different distances and the respective pixels were measured (Fig.14).

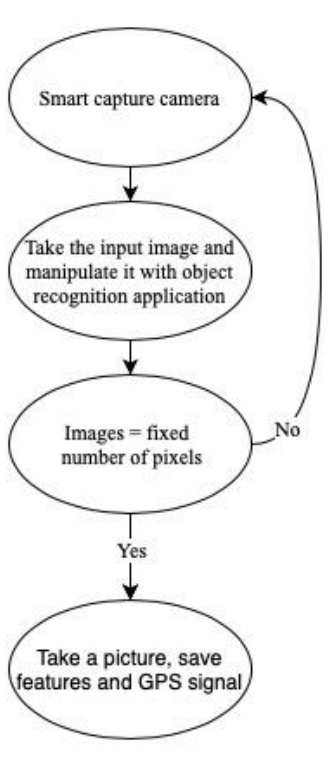

Fig.14. Flow chart of frame acquisition from smart cam.

\subsection{Mathematical model of georeferencing frames}

The mathematical model of georeferencing used is derived from the El-Sheimy and Schwarz 2004 formula:

$r_{p}^{m}=r_{I M U}^{m}(t)+R_{I M U}^{m}(t) \cdot\left[s_{p} \cdot R_{s}^{I M U} \cdot r_{p}^{s}+r_{I M U / s}^{I M U}\right]$

(6)

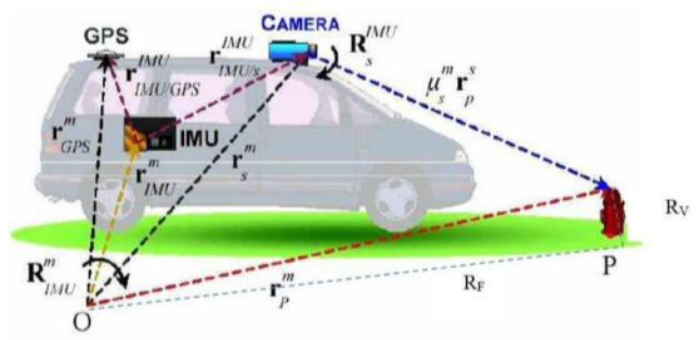

\begin{tabular}{|c|c|c|}
\hline$r_{p}^{m}$ & $\begin{array}{c}\text { Vector position point } \mathrm{P} \text { in the mapping } \\
\text { frame }\end{array}$ & Unknow \\
\hline$r_{I M U}^{m}(t)$ & $\begin{array}{c}\text { Original position of the sensor in the } \\
\text { mapping frame at time t }\end{array}$ & From GPS/INS \\
\hline$R_{I M U}^{m}(t)$ & $\begin{array}{c}\text { Rotation matrix from the IMU body frame } \\
\text { to mapping frame at time }\end{array}$ & From GPS/INS \\
\hline$S_{p}$ & $\begin{array}{c}\text { Ratio between the camera-point distance and } \\
\text { the vector } r_{p}^{s} \text { lenght }\end{array}$ & From restitution \\
\hline$R_{S}^{I M U}$ & $\begin{array}{c}\text { Rotation matrix between camera frame and } \\
\text { body frame }\end{array}$ & $\begin{array}{c}\text { Calibration of shooting } \\
\text { system }\end{array}$ \\
\hline$r_{p}^{S}$ & $\begin{array}{c}\text { Vector of the image coordinates (x, y, -c) of } \\
\text { the point } \mathrm{P}\end{array}$ & $\begin{array}{c}\text { Measured } \\
\text { frame }\end{array}$ \\
\hline$r_{I M U / S}^{I M U}$ & Vector position of the body & $\begin{array}{c}\text { Calibration of shooting } \\
\text { system }\end{array}$ \\
\hline
\end{tabular}

Fig. 15. Georeferencing vector calculation scheme

Applying the projection on the street level of the positioning to the recognition of the pattern: 


$$
\mathrm{R}_{\mathrm{F}}=r_{p}^{m}+\mathrm{R}_{\mathrm{v}}
$$

The value of the $\mathrm{Rv}$ vector is determined in relation to the position of the bounding box of the signal in the frame with respect to the progress of the MMS. The dimensions of the signal are known according to the type of road in which it is installed.

\subsection{Data Localization Crack GIS (DLCGIS)}

For the location of cracks, horizontal and vertical road signs and manholes, an automatic procedure called Data Localization GIS (DLCGIS) has been implemented which provides the signaling of the presence of cracks, horizontal and vertical road signs and manholes within the GIS using artificial intelligence algorithms, used after a phase of identification of the elements of interest, to automatically store the position of the objects and their alphanumeric data. Then follows the subsequent export of the data acquired within the GIS, where the "historical" update is managed in the existing database, in order to verify the effectiveness of the development of the infrastructure.

Once the various cracks, horizontal and vertical road signs and manholes have been identified, they are loaded into a special layer, and "snapped" (moved and anchored) with any polyline present in the layer where the road cartography is represented $[21 ; 22]$.

Specifically, the used procedure works as summarized in the Fig. $16-21$.

In more detail, the DLCGIS was developed in four software modules (Fig. 16 - 21), each with specific functions:

1) The Plug in Module extends the number of objects that can be represented recognizable and classifiable;

2) The kernel, that interact with the users, coordinates the different modules, pre-processing and postprocessing the Input/Output data of the modules themselves;

3) The NNS (Neural Network System) module processes the algorithm implementing the Neural Network trained to recognize the elements of interest;

4) The GIS I/O (Input/Output) Module manages the interface with the GIS software.

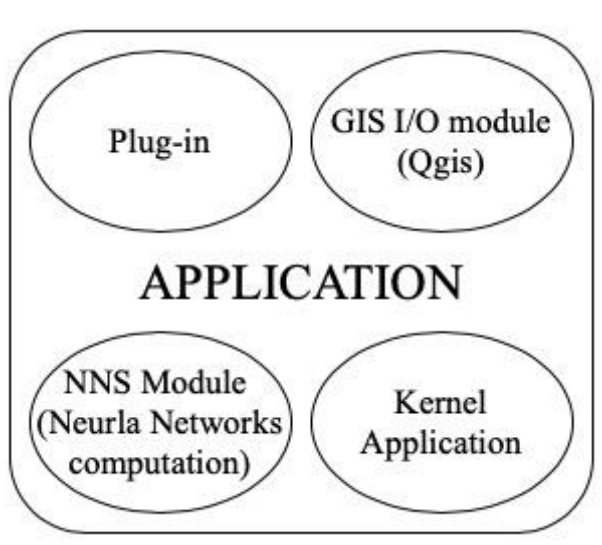

Fig. 16. Schematic representation of the four modules that compose the automatic cracks, horizontal and vertical road signs and manholes classification application.

An example of GIS Input/Output module is shown in Fig. 17. In particular, the GIS I/O module is given in Input the files (polylines and polygons) in shp-dbf format (representatives of the road route), returning to Output a space database where the different attributes have been assigned to the objects.

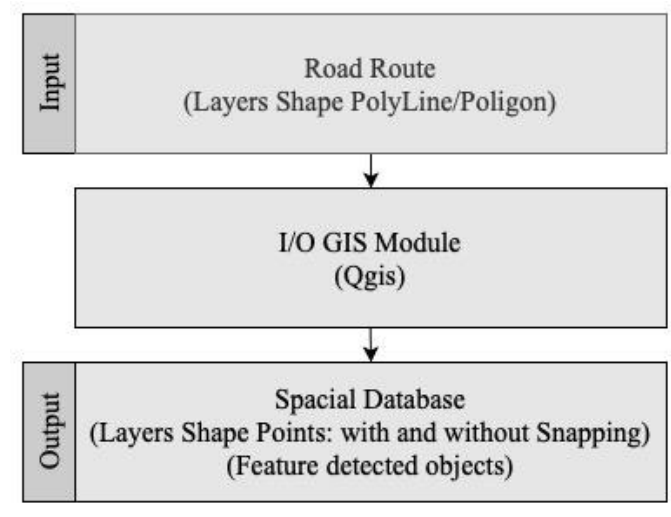

Fig. 17. The GIS I /O module interfaces with the GIS software using files in shp-dbf format.

The NNS (Neural Network System) module instead processes the algorithm implementing the Neural Network trained to recognize the elements of interest; in particular, the image is portioned and processed by different neural networks. The NNS manages the various results of the same in order to recognize and identify the contours of the object being investigated (Fig. 18). 


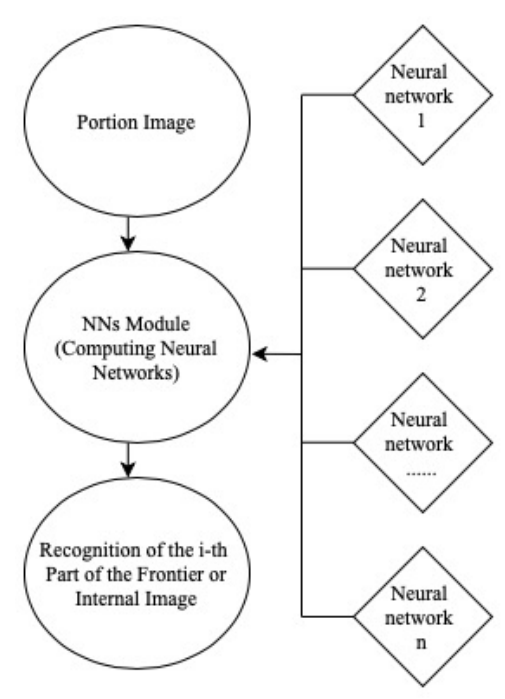

Fig. 18. The NNS Module computes the different models of the Neural Network.

Subsequently, the Plug in Module extends the number of objects that can be represented, recognized and classified, assigning and identifying within the parameter processing range the type of border, the different shades of depth color (Fig.19).

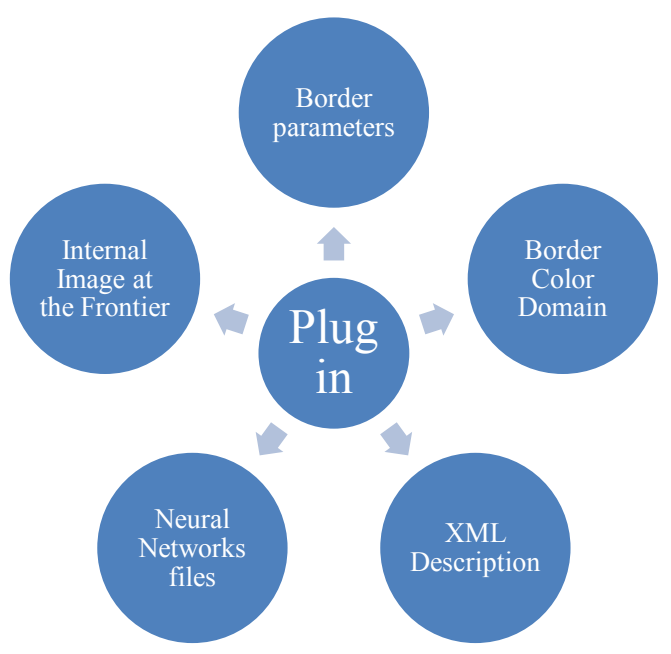

Fig. 19. A Plug-In valid for the application and the parameters that make it up.

The kernel, that interact with the users, coordinates the different modules, pre-processing and postprocessing the Input/Output data of the modules themselves.

The application Kernel works with spatial adjustments (Simple and Intelligent Snapping) on the detected and recognized elements. Through this module the Kernel exports to the GIS one or more specific layers containing the spatial and alphanumeric data (type of signal, etc.) of the identified elements; the data refer to both "adjusted" and unadjusted elements.

The system developed for the study area has been tested on additional test areas as shown in fig. 20 21 , and allows, selected a graph, to identify the presence or absence of discrepancies in the roadway (as shown in the figure 20) with a different color (red) when the roadway affected by the presence of the holes is greater than $25 \%$ of the site area road itself or. It also allows you to identify the geolocation of vertical signs and manholes (Fig. 21), of horizontal signs and their degree of deterioration. The generated database can be consulted.

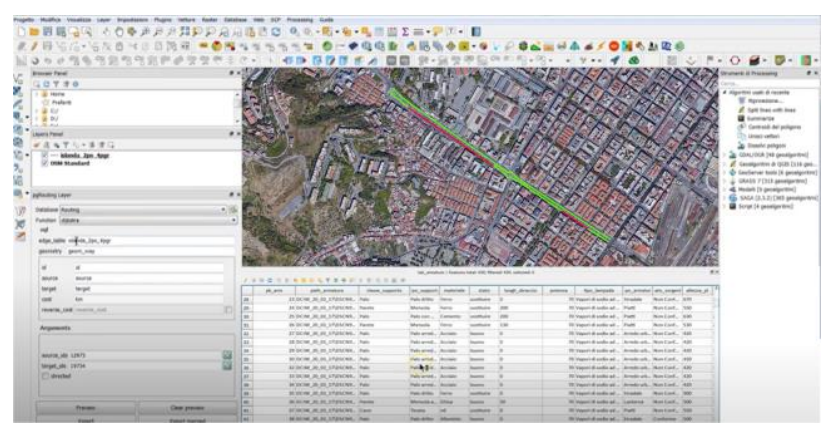

Fig. 20. Display on the screen of the properties of the road graph. In red the section with deterioration above $25 \%$.

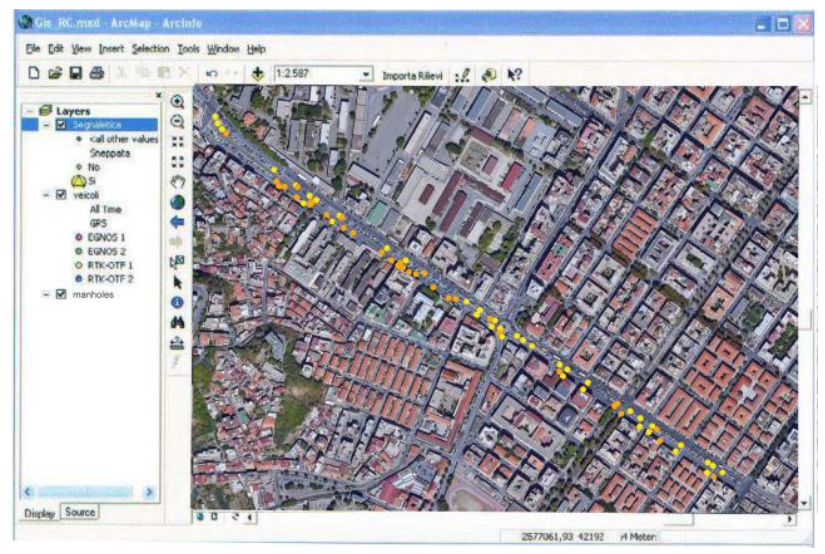

Fig. 20. Identification of vertical road signs (yellow) and manholes (orange) within the software.

\section{Discussion}

There are different methods and tools for updating the road cadastre, each has its strengths and weaknesses. The solution proposed by us tries to overcome these defects and propose the best solution for updating the road cadastre.

Airborne laser scanning (ALS) is currently widely applied for country wide DTM generation [23], and corridor mapping (mapping of railroads, streets and 
highways, and riversides) [24]. ALS is due to transfer costs, multitemporal laser surveys (including change detection, e.g.) are seldom studied and developed. A summary of ALS can be found in [25] and in [26]. The state-of-the-art in multitemporal ALS research can be found in brief from [27 - 30].

The development of change detection processes is hindered by the lack of good quality data, especially together with hyperspectral images, is expensive due to transfer costs.

Relating the application developed by us with what is reported in the bibliography, the further reduction of costs for the acquisition and management of the data acquired by us is quite evident, being able to reduce the times by $1 / 3$ compared to the alternatives.

Among the alternatives to the UAV for road surface monitoring we can use the mobile mapping system (MMS).

An MMS consists of the integration of three main hardware components: optical sensors (laser scanner and/or digital cameras), navigation/positioning sensors (IMU/GNSS) and a control and synchronization unit.

Mobile mapping systems using LiDAR technology (also called Mobile Laser Scanning - MLS systems) are the most recent solution. They acquire dense point clouds in real time and offer important advantages, such as: high data acquisition speed, with consequent reduction in costs and time required; high spatial density of the measured data, which allows a dense 3D reconstruction of the detected scene; remote data acquisition, which guarantees the efficiency and safety of the survey $[31 ; 32 ; 33]$.

MMS has multiple advantages, however the processing and management of the data collected, and the use of multiple operators often discourages possible users and entails a higher cost than the solution we propose.

In fact, the use of a monitoring solution based on drones allows to automate the monitoring processes eliminating the need to employ more operators, reduces the risk of accidents due to other road vehicles and allows to reach points otherwise inaccessible with an MMS or with other tools.

Furthermore, we carried out a survey (which will be the subject of future papers) on a viaduct both with an MMS and with the UAV system proposed by us. The results obtained verify the effective convenience of times and costs of the system we propose compared to the classic MMS.

Using our proposed and developed system (Fig.13), we automatize both the data acquisition phase, both the data transmission phase and the GIS update phase. We can program and control a fleet of automated drones connected to the cloud (or a local network) that are automatically recharged through special charging stations located in pre-established points. These drones rise in flight and acquired data, that are transmitted in a cloud platform (in this way the drone fleet provides a real-time data feedback, that translates into a faster analysis of the acquired data, providing immediate results compared to conventional methods).

Data processing is done automatically directly in the cloud and finally DLCGIS was created as regards the GIS update phase.

This procedure allows us to reduce time and costs and increase efficiency.

In the future, the next challenge in the field of scientific research to the automation of UAV monitoring will be - the logical treatment of the collected data - improvement of the automation data processing - improvement of precision quality in the automatic selection of data.

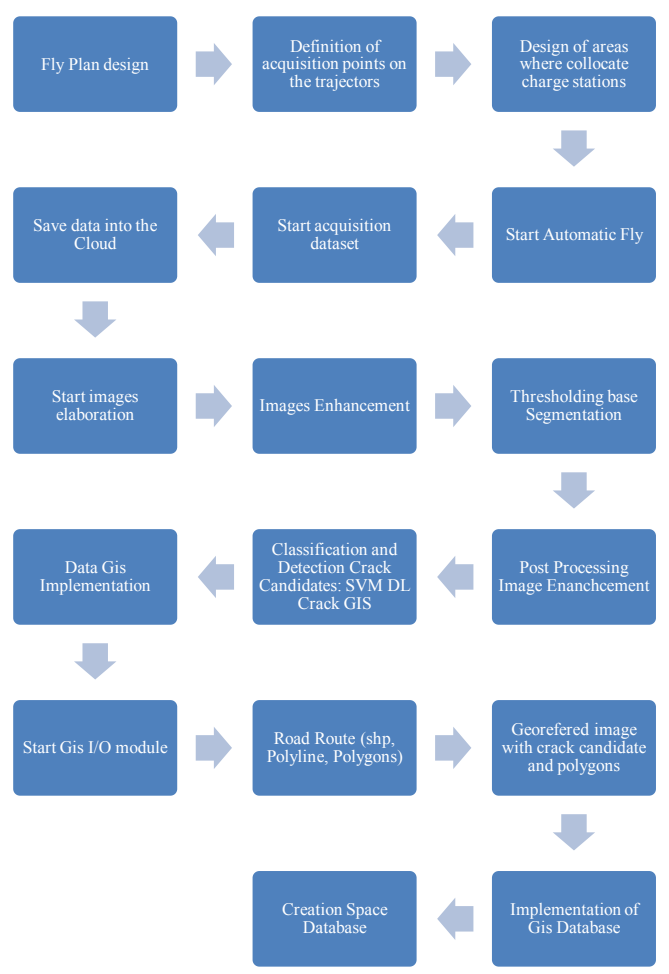

Fig. 21. New methodology flowchart

\section{Conclusions}

The incidence of road pavement degradation and vertical and horizontal signs is now the cause of numerous accidents. The proposed methodology for the automatic survey can be included for the inspections of road infrastructure and therefore allows who in charge (in the public institution) to be able to plan interventions.

From an economic point of view, the monitoring system proposed by us is economically more 
advantageous than other solutions that provide similar results; and therefore, if implemented with roadside maintenance functions, this can be an excellent tool for management agencies, which can therefore schedule maintenance based on GIS indications.

The advantages are enormous: economic savings; time saving; greater safety for operators who during the monitoring phase will not have to physically go to the site, thus avoiding being involved in possible road accidents.

The automation of the process allows you to easily identify the areas to be repaired but also to identify over time the areas most subject to wear and tear (areas subject to greater traffic, atmospheric agents, heavy transport, etc.) and therefore improve the programming of the maintenance and resource management.

The Geomatics Laboratory of the Mediterranea University has developed a prototype system for the continuous survey of the state of road paving, horizontal and vertical road signs and manholes.

The product system, still in progress, sends georeferred dataset to a processing system that through classification algorithms already today allows the correct detection of the same dataset with a very satisfying result.

In the future we plan to extend the system, currently limited to the study area only.

Although the National Civil Aviation Authority (ENAC) and the European Union Aviation Safety Agency (EASA) have recently significantly expanded the scope of use of drones, one of the biggest limitations remains the obligation not to fly overcrowded areas and obligation to use the operator. In this regard, we propose to install parachute systems in drones in order to avoid damage to things and people in case of unexpected events.

In addition, it would be advisable to provide, under the drone's trajectory, a buffer zone in order to warn the users that pass through it to be in an area possibly flying over drones.

\section{Reference}

[1] S. Ogawa, K. Matsushima and O. Takahashi, Efficient Pavement Crack Area Classification Using Gaussian Mixture Model Based Features, International Conference on Mechatronics, Robotics and Systems Engineering (MoRSE), Bali, Indonesia, 2019, pp. 75-80.

Doi: 10.1109/MoRSE48060.2019.8998713

[2] T. N. Tonkin and N. G. Midgley, GroundControl Networks for Image Based Surface Reconstruction: An Investigation of Optimum
Survey Designs Using UAV Derived Imagery and Structure-from-Motion Photogrammetry, Remote Sens., Vol.8, Issue 9, 2016, pp. 786. Doi: https://doi.org/10.3390/rs8090786

[3] B. Sekeroglu, K. Tuncal, Image Processing in Unmanned Aerial Vehicles, Al-Turjman F. (eds) Unmanned Aerial Vehicles in Smart Cities. Unmanned System Technologies, Springer, Cham, 2020, pp. 167-179.

Doi: https://doi.org/10.1007/978-3-030-38712$9+10$

[4] F. Dadrasjavan, N. Zarrinpanjeh, A. Ameri, Automatic Crack Detection of Road Pavement Based on Aerial UAV Imagery, Preprints, 2019, 2019070009.

Doi: 10.20944/preprints201907.0009.v1

[5] S. Chen, L. Truong-Hong, D. F. Laefer, E. Mangina, (2018), Automated Bridge Deck Evaluation through UAV Derived Point Cloud, CERI-ITRN2018, Dublin, Ireland, 2018, pp. 735-740.

[6] N. Hoang, Q. Nguyen, A novel method for asphalt pavement crack classification based on image processing and machine learning, Engineering with Computers, Vol. 35, 2019, pp. 487-498.

Doi: https://doi.org/10.1007/s00366-018-06119

[7] Y. Sari, P. B. Prakoso and A. R. Baskara, Road Crack Detection using Support Vector Machine (SVM) and OTSU Algorithm, 6th International Conference on Electric Vehicular Technology (ICEVT), Bali, Indonesia, 2019, pp. 349-354. Doi: 10.1109/ICEVT48285.2019.8993969

[8] A. Ameri, F. Dadrass Javan, N. Zarrinpanjeh, Automatic Pavement Crack Detection Based on Aerial Imagery, Journal of Geomatics Science And Technology 2019, Vol.9, No.1 \#a00641. pp. $145-160$.

[9] C. Chen, H. S. Seo, Y. Zhao, B. Chen, J.W. Kim, Y. Choi, and M. Bang, Automatic Pavement Crack Detection Based on Image Recognition, International Conference on Smart Infrastructure and Construction, (ICSIC), 2019, pp 361-369.

Doi: https://doi.org/10.1680/icsic.64669.361

[10] I.L. Al-Qadi, M. Elseifi, and P.J. Yoo, In-situ validation of mechanistic pavement finite element modeling, 2nd Int. Conf. on Accelerated Pavement Testing 1, CD-ROM, 2004.

[11] S. Erlingsson, 3-D FE analyses of HVS tested low volume road structures - comparison with measurements, Proc. 3rd Int. Symp. on 3D Finite Element for Pavement Analysis, Design \& Research, Vol.1, 2002, pp. 339-350. 
[12] M.A. Elseifi, I.L. Al-Qadi, and P.J. Yoo, Viscoelastic Modeling and Field Validation of Flexible Pavements, J. Engineering Mechanics, Vol.132, Issue 2, 2006.

Doi: $\quad$ https://doi.org/10.1061/(ASCE)07339399(2006)132:2(172)

[13] H. Yin, S. Stoffels, and M. Solaimanian, Optimization of Asphalt Pavement Modeling based on the Global-Local 3D FEM Approach, Road Materials and Pavement Design, Vol.9, Issue 2, 2008, pp. 345-355.

Doi:https://doi.org/10.1080/14680629.2008.96 90122

[14] M. A. Onyango, Verification of mechanistic prediction models for permanent deformation in asphalt mixes using accelerated pavement testing, PHD Dissertation, Kansas State University, Kansas, 2009.

[15] I. L. Al-Qadi, P.J. Yoo, M.A. Elseifi, and S. Nelson, Creep Behavior of Hot-Mix Asphalt due to Heavy Vehicular Tire Loading, J.of Engineering Mechanics, Vol.135, Issue 11, 2009, pp. 1265-1273.

Doi: $\quad$ https://doi.org/10.1061/(ASCE)07339399(2009)135:11(1265)

[16] M. Kim, Three-dimensional finite element analysis of flexible pavements considering nonlinear pavement foundation behavior, $P h D$ Thesis, University of Illinois, Urbana, 2007.

[17] C. Huang, R. Abu Al-Rub, E. Masad, and D. Little, Threedimensional simulations of asphalt pavement permanent deformation using a nonlinear viscoelastic and viscoplastic model, $J$. Materials in Civil Engineering, Vol. 23, 2011, pp. 56-68.

[18] L. A. Al-Khateeb, A. Saoud, and M. F. AlMsouti, Rutting Prediction of Flexible Pavements Using Finite Element Modeling, Jordan J. Civil Engineering, Vol.5, No.2, 2011, pp. 173-190.

[19] S. Pandey, K. R. Rao, and D. Tiwari, Effect of geogrid reinforcement on critical responses of bituminous pavements, 25th ARRB Conf., 2012.

[20] Y. Sari, P. B. Prakoso, A. R. Baskara, Application of neural network method for road crack detection, TELKOMNIKA, Telecommunication, Computing, Electronics and Control, Vol.18, No.4, 2020, pp. 19621967.

Doi: 10.12928/TELKOMNIKA.v18i4.14825

[21] V. Barrile, F. Cotroneo, F. Praticò, Automatic updating processes of road surface surveys and surface defects: proposal of an innovative highperformance method, SIIV National Conference Cosenza, Italy, 2006.
[22] V. Barrile, F. Cotroneo, A software for the automatic update of the road cadastre in the GIS environment, Bulletin of the Italian society of photogrammetry and topography, 2006, pp. 923.

[23] G. Sithole, G. Vosselman, Experimental comparison of filter algorithms for bare-earth extraction from airborne laser scanning point clouds, ISPRS Journal of Photogrammetry and Remote Sensing, 2004, Vol.59, Issues 1-2, pp. 85-101.

Doi:https://doi.org/10.1016/j.isprsjprs.2004.05. 004

[24] B. Höfle, M. Vetter, N. Pfeifer, G. Mandlburger, J. Stötter, Water surface mapping from airborne laser scanning using signal intensity and elevation data, Earth Surface Processes and Landforms, 2009, Vol.34, Issue 12, pp. 16351649 .

Doi: https://doi.org/10.1002/esp.1853

[25] J. Shan, C. K. Toth, Topographic Laser Ranging and Scanning: Principles and Processing, Second Edition. CRC Press, Taylor \& Francis Group, 2018.

[26] G. Vosselman, H. G. Maas, Airborne and Terrestrial Laser Scanning, Whittles Publishing, Caithes, GB, 2010, p. 336, ISBN: 978-1904445-87-6.

[27] S. Solberg, E. Naesset, K. Holt Hanssen, E. Christiansen, Mapping defoliation during a severe insect attack on Scots pine using airborne laser scanning, Remote Sensing of Environment, Vol. 102, Issue 3-4, 2006, pp. 364-376.

Doi: https://doi.org/10.1016/j.rse.2006.03.001

[28] T. Vögtle, E. Steinle, Detection and recognition of changes in building geometry derived from multitemporal laserscanning data. International Archives of Photogrammetry, Remote Sensing and Spatial Information Sciences, 35 (Part B2), 2004, pp. 428-433.

[29] X. Yu, J. Hyypp, H. Kaartinen, H. Hyyppä, M. Maltamo, P. Rönnholm, Measuring the growth of individual trees using multitemporal airborne laser scanning point clouds. International Archives of Photogrammetry, Remote Sensing and Spatial Information Sciences, Vol. XXXVI3/W19, WG III/3, III/4, V/3, 2005, pp. 204-208.

[30] X. Yu, J. Hyypp, H. Kaartinen, M. Maltamo, Automatic detection of harvested trees and determination of forest growth using airborne laser scanning. Remote Sensing of Environment, Vol.90, Issue 4, 2004, pp. 451-462.

Doi: https://doi.org/10.1016/j.rse.2004.02.001

[31] V. Barrile, G. Leonardi, A. Fotia, G. Bilotta, G. Ielo, Real-Time Update of the Road Cadastre in 
GIS Environment from a MMS Rudimentary System, International Symposium on New Metropolitan Perspectives, Springer, Cham, Vol. 101, 2018, pp. 240-247.

Doi: $\quad$ https://doi.org/10.1007/978-3-31992102-0 26

[32] A. Mancini, E. S. Malinverni, E. Frontoni, P. Zingaretti, Road pavement crack automatic detection by MMS images, 21st Mediterranean Conference on Control and Automation, 21st Mediterranean Conference on Control and Automation, Chania, 2013, pp. 1589-1596, Doi: 10.1109/MED.2013.6608934

[33] Z. Florkova, L. Duris, M. Veselovsky, S. Sedivý and D. Kovalova, Three-dimensional mobile mapping system and its use in road engineering, MATEC Web Conf., Vol. 196, No.04082, 2018, Doi:https://doi.org/10.1051/matecconf/2018196 04082

[34] V. Barrile, E. Bernardo, A. Fotia, G. Candela, G. Bilotta, Road Safety: Road Degradation Survey Through Images by UAV. WSEAS TRANSACTIONS on ENVIRONMENT and DEVELOPMENT.
Creative Commons Attribution License 4.0 (Attribution 4.0 International, CC BY 4.0)

This article is published under the terms of the Creative Commons Attribution License 4.0 https://creativecommons.org/licenses/by/4.0/deed.en_US 